\title{
Nonreciprocal vibrations of finite elastic structures with spatiotemporally modulated material properties
}

\author{
Benjamin M. Goldsberry ${ }^{\circ},{ }^{1}$ Samuel P. Wallen ${ }^{1},{ }^{1,2}$ and Michael R. Haberman ${ }^{1,2}$ \\ ${ }^{1}$ Applied Research Laboratories, The University of Texas at Austin, Austin, Texas 78758, USA \\ ${ }^{2}$ Walker Department of Mechanical Engineering, The University of Texas at Austin, Austin, Texas 78712-1591, USA
}

(Received 27 March 2020; revised 27 May 2020; accepted 23 June 2020; published 23 July 2020)

\begin{abstract}
Elastic waveguides with time- and space-dependent material properties have received great attention as a means to realize nonreciprocal propagation of small-amplitude mechanical waves in unbounded elastic media. Previous works have shown that propagating waves in a modulated medium violate reciprocity by means of asymmetric frequency and wave number conversion between two counterpropagating modes. In the present study, we investigate nonreciprocal longitudinal and transverse vibrations in a finite elastic waveguide with time- and space-dependent material properties. A semianalytical approach based on coupled mode theory is derived, which makes use of the mode shapes of the nonmodulated beam subject to a set of imposed boundary conditions. The modulation parameter space is explored for designs that yield a large degree of nonreciprocity for low-frequency longitudinal and transverse vibrations. For the cases considered in this work, only a small subset of modulation parameters displays strong nonreciprocity, which reveals a sparse and complex design space that must be analyzed in order to create nonreciprocal wave devices.
\end{abstract}

DOI: 10.1103/PhysRevB.102.014312

\section{INTRODUCTION}

Acoustic and elastic wave reciprocity is an important fundamental property that details the characteristics of wave propagation between two points. Namely, in a heterogeneous linear time-invariant (LTI) medium, the propagation of acoustic or elastic waves from source to receiver will be identical in phase and magnitude if the source and receiver are interchanged [1-3]. Breaking reciprocity can enable unique wave phenomena, such as direction-dependent frequency [4] and mode conversion [5], which yields directional band gaps $[6,7]$ for propagating waves. These effects may be exploited to design novel devices, including acoustic circulators $[8,9]$ and topological insulators $[10,11]$, which may be used to improve energy harvesting, signal processing, vibration isolation, and acoustic communication devices.

One key concept of realizing a nonreciprocal LTI system is to violate time-reversal symmetry. This was initially achieved by introducing a directional momentum bias for mechanical systems, such as fluid flow $[8,12,13]$, but was later demonstrated in piezophononic media [14], moving media $[8,15,16]$, gyroscopic phononic crystals $[17,18]$, and media with modulated resonators $[19,20]$, for example. The principle of reciprocity can also be violated in media that are not LTI. This includes nonlinear media, which have been used to create one-way sound propagation in systems with geometric and material stiffness nonlinearities, internal scale hierarchy, and materials composed of unit cells with geometric asymmetry

Published by the American Physical Society under the terms of the Creative Commons Attribution 4.0 International license. Further distribution of this work must maintain attribution to the author(s) and the published article's title, journal citation, and DOI.
[21-24]. Another mechanism, which is central to the present study, is modulation of the material properties in space and time $[6,7,25-30]$. Nonreciprocal wave propagation has been observed in media whose effective mechanical properties have been externally modulated using electromagnetic effects [31-34], as well as external mechanical deformation via "small-on-large" wave propagation in discrete [35] and continuous [36] chains of geometrically nonlinear elements.

Although much research considers wave motion in infinite media with spatiotemporal modulation of properties, properly accounting for wave propagation in structures constructed from constituents with time- and space-varying properties introduces additional complexities. More specifically, the majority of current research has investigated only nonreciprocal plane wave propagation in infinitely periodic simple systems, such as one-dimensional mass-spring chains [27], longitudinal and flexural waves in beams [6], locally resonant metamaterials [7,37], and two-dimensional systems such as membranes [38] and plates [39]. Nonreciprocity is demonstrated in these works via the existence of directional band gaps in the frequency-wave-number dispersion spectrum. However, these concepts are difficult to apply to a bounded medium due to the direction-dependent frequency conversion present when each plane wave component of the total wave field is reflected from the boundaries. A formulation that exactly incorporates the boundary conditions, frequency conversions, and standing wave patterns for steady-state vibrations is therefore needed in order to properly design finite domains and structures whose properties are modulated in time and space.

In this work, we study longitudinal and transverse modes in a finite elastic Euler-Bernoulli beam in which Young's modulus is modulated in space and time [6,19,40,41]. A coupled-mode approach is utilized by representing the total displacement solution as a weighted sum of modes that 

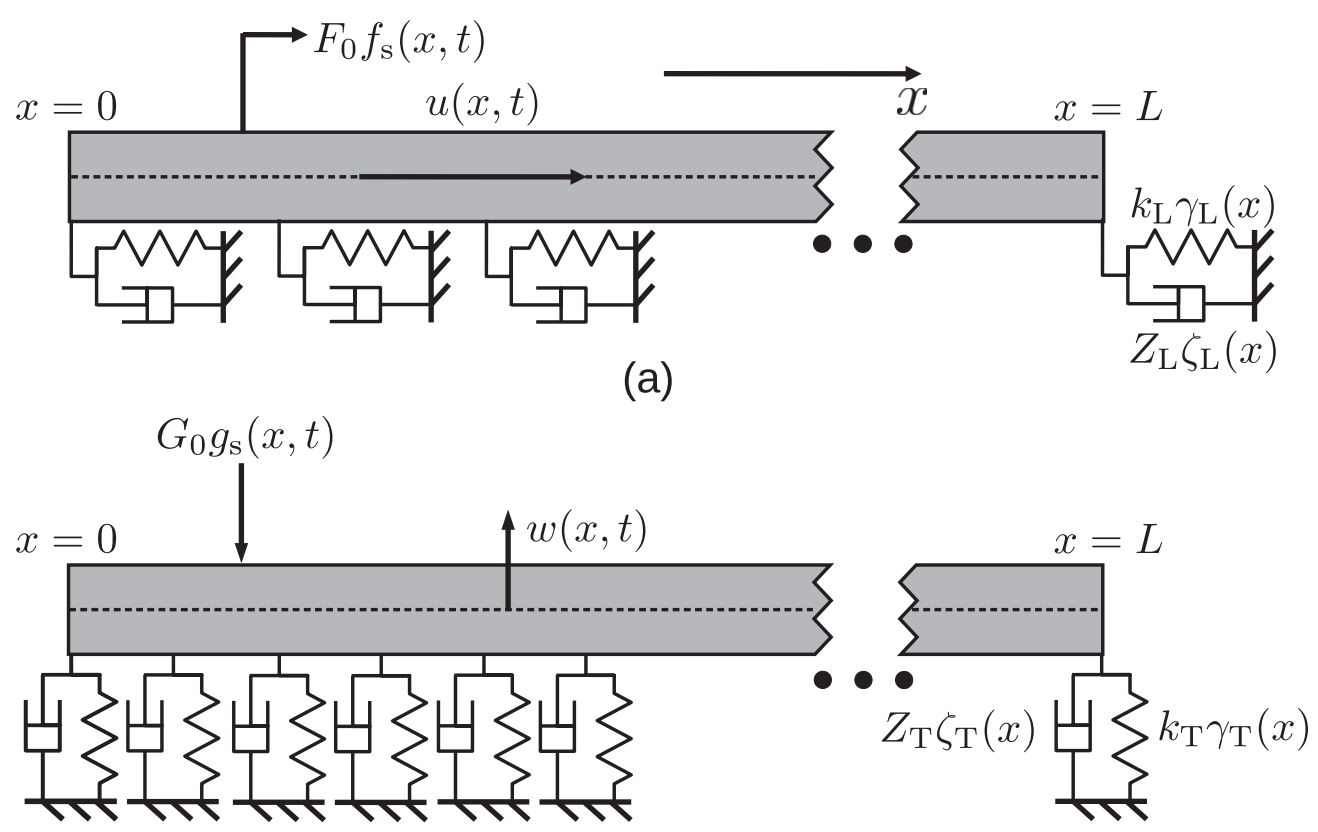

(b)

FIG. 1. Schematic of the Euler-Bernoulli beam of length $L$. (a) Longitudinal wave configuration with fixed or free boundary conditions applied at the ends of the beam. (b) Transverse wave configuration with fixed, pinned, free, or roller boundary conditions applied at the ends of the beam. Both configurations include a continuous distribution of external reactive (elastic) and resistive (viscous) forces, represented above by springs and dashpots.

are known a priori from the nonmodulated beam case. We make no assumptions on the depth of modulation of Young's modulus relative to its mean value as well as the modulation frequency relative to the drive frequency, which differs from some previous works that required small modulation depths and frequencies to enable perturbative approaches [7,9] and allows for an extensive exploration of the design space. We then seek material modulation parameters that yield a high degree of nonreciprocity between a point force source and receiver along the beam. This work shows that the spatiotemporal modulation of the material properties alone does not guarantee strong nonreciprocity in finite continuous systems, but instead introduces a sparse and complex design space that must be thoroughly explored in order to create nonreciprocal wave devices.

\section{THEORETICAL MODEL}

Consider longitudinal and transverse wave propagation in a thin elastic Euler-Bernoulli beam of length $L$ with constant cross-sectional area $A_{0}$ and constant second moment of inertia $I$. The dynamic longitudinal displacement $u(x, t)$ and transverse displacement $w(x, t)$ of the beam are uncoupled for this case and are described by their respective partial differential equations (PDEs),

$$
\begin{gathered}
\frac{\partial}{\partial x}\left(E(x, t) \frac{\partial u}{\partial x}\right)-\rho_{0} \frac{\partial^{2} u}{\partial t^{2}}=f\left(x, t, u, \frac{\partial u}{\partial t}\right), \\
R_{g}^{2} \frac{\partial^{2}}{\partial x^{2}}\left(E(x, t) \frac{\partial^{2} w}{\partial x^{2}}\right)+\rho_{0} \frac{\partial^{2} w}{\partial t^{2}}=g\left(x, t, w, \frac{\partial w}{\partial t}\right),
\end{gathered}
$$

where $E(x, t)$ is Young's modulus, $\rho_{0}$ is the density, $R_{g}=$ $\sqrt{I / A_{0}}$ is the radius of gyration of the beam, and $f\left(x, t, u, \frac{\partial u}{\partial t}\right)$ and $g\left(x, t, w, \frac{\partial w}{\partial t}\right)$ are the exterior forcing per unit volume in the $x$ direction and orthogonal to the $x$ direction, respectively, which can contain linear reactive (elastic) and/or resistive (viscous) forces, as well as prescribed driving forces. Only homogeneous boundary conditions are considered in this work, which includes fixed or free boundaries for the longitudinal wave case and clamped, pinned, roller, or free boundaries for the transverse wave case.

The geometry is shown in Fig. 1(a) for the longitudinal wave case and Fig. 1(b) for the transverse wave case. In both cases we assume a continuous exterior distribution of elastic and viscous forces, such that the exterior forcing functions for longitudinal and transverse waves can be written as

$$
\begin{gathered}
f\left(x, t, u, \frac{\partial u}{\partial t}\right)=\frac{F_{0}}{A_{0}} f_{\mathrm{s}}(x, t)+\frac{k_{\mathrm{L}}}{A_{0}} \gamma_{\mathrm{L}}(x) u+\frac{Z_{\mathrm{L}}}{A_{0}} \zeta_{\mathrm{L}}(x) \frac{\partial u}{\partial t}, \\
g\left(x, t, w, \frac{\partial w}{\partial t}\right)=\frac{G_{0}}{A_{0}} g_{\mathrm{s}}(x, t)+\frac{k_{\mathrm{T}}}{A_{0}} \gamma_{\mathrm{T}}(x) u+\frac{Z_{\mathrm{T}}}{A_{0}} \zeta_{\mathrm{T}}(x) \frac{\partial w}{\partial t},
\end{gathered}
$$

where $F_{0}\left(G_{0}\right)$ is the exterior force amplitude and $f_{\mathrm{s}}(x, t)$ $\left(g_{\mathrm{s}}(x, t)\right)$ is the exterior force density per unit length for the longitudinal (transverse) case, $k$ is the exterior elastic stiffness with spatial density $\gamma(x)$ per unit length, $Z$ is the mechanical impedance of the resistive forces with spatial density $\zeta(x)$ per unit length, and the subscripts $\mathrm{L}$ and $\mathrm{T}$ refer to the longitudinal and transverse wave cases, respectively. 
We further assume that Young's modulus has the form

$$
E(x, t)=E_{0}+E_{\mathrm{m}} \chi(x, t),
$$

where $E_{0}$ is the mean (static) Young's modulus, $E_{\mathrm{m}}$ is the modulation amplitude, and $\chi(x, t)$ is the variation, or modulation, of Young's modulus about the mean value. Finally, we restrict $\chi(x, t)$ to be a periodic function of time,

$$
\chi(x, t)=\chi\left(x, t+T_{\mathrm{m}}\right),
$$

where $T_{\mathrm{m}}$ is the characteristic modulation time and $\omega_{\mathrm{m}}=$ $2 \pi / T_{\mathrm{m}}$ is the modulation angular frequency.

In order to solve Eqs. (1) and (2), we require a set of basis functions to represent the solution. However, the choice of basis functions is nontrivial when material properties are modulated in space and time since the separation by variables method cannot be utilized. We therefore choose to use the mode shapes of the nonmodulated beam that satisfy the given boundary conditions as a basis set due to its simple analytical form and the fact that they form a complete orthogonal set. The spatiotemporal modulation of the material properties along with the exterior elastic and viscous forces will then introduce coupling between the modes of the nonmodulated beam. In the following section, we derive the coupled-mode formulation for the longitudinal modes, followed by the transverse mode case.

\section{A. Longitudinal modes}

To solve Eq. (1), we seek a solution of the form

$$
u(x, t)=\sum_{i=1}^{\infty} u_{i}(t) \psi_{i}(x),
$$

where $u_{i}(t)$ are the time-dependent modal amplitudes, and $\psi_{i}(x)$ are the mode shapes that satisfy the homogeneous constant-coefficient Helmholtz equation provided in Eq. (8) subject to the boundary conditions imposed at the ends of the beam,

$$
E_{0} \frac{d^{2} \psi_{i}}{d x^{2}}+\rho_{0} \omega_{i}^{2} \psi_{i}=0,
$$

where $\omega_{i}$ are the natural frequencies of the modes. Consequently, the modes satisfy the orthogonality relation

$$
\int_{0}^{L} \psi_{i} \psi_{j} d x= \begin{cases}0 & \text { if } i \neq j, \\ \beta & \text { if } i=j,\end{cases}
$$

where $\beta$ is the mode normalization constant [42]. Substitution of Eq. (7) into Eq. (1) gives the following expression for forced longitudinal wave motion in the beam:

$$
\begin{aligned}
& \sum_{i=1}^{\infty}\left[u_{i}(t) \frac{\partial}{\partial x}\left(E(x, t) \frac{\partial \psi_{i}}{\partial x}\right)-\rho_{0} \frac{\partial^{2} u_{i}}{\partial t^{2}} \psi_{i}\right] \\
& =f\left(x, t, u, \frac{\partial u}{\partial t}\right) .
\end{aligned}
$$

Multiplying Eq. (10) on both sides by $\psi_{j}$, integrating the expression over the length of the beam $L$, and utilizing the orthogonality of the modes, Eq. (9), yields the following system of coupled ordinary differential equations (ODEs) for the modal amplitudes:

$$
\begin{aligned}
\rho_{0} \beta \frac{\partial^{2} u_{i}}{\partial t^{2}}= & \sum_{j=1}^{\infty} u_{j}(t) \int_{0}^{L} \frac{\partial}{\partial x}\left(E(x, t) \frac{\partial \psi_{j}}{\partial x}\right) \psi_{i} d x \\
& -\int_{0}^{L} f\left(x, t, u, \frac{\partial u}{\partial t}\right) \psi_{i} d x .
\end{aligned}
$$

The first integral on the right-hand side of Eq. (11) is rewritten using integration by parts:

$$
\begin{aligned}
\int_{0}^{L} \frac{\partial}{\partial x}\left(E(x, t) \frac{\partial \psi_{j}}{\partial x}\right) \psi_{i} d x= & \left.E(x, t) \frac{\partial \psi_{j}}{\partial x} \psi_{i}\right|_{0} ^{L} \\
& -\int_{0}^{L} E(x, t) \frac{\partial \psi_{i}}{\partial x} \frac{\partial \psi_{j}}{\partial x} d x .
\end{aligned}
$$

Note that the boundary terms in Eq. (12) vanish if the normal modes satisfy clamped (Dirichlet) or free (Neumann) boundary conditions. For these cases, Eq. (11) reduces to the expression

$$
\begin{aligned}
\rho_{0} \beta \frac{\partial^{2} u_{i}}{\partial t^{2}}= & -\sum_{j=1}^{\infty} u_{j} \int_{0}^{L} E(x, t) \frac{\partial \psi_{i}}{\partial x} \frac{\partial \psi_{j}}{\partial x} d x \\
& -\int_{0}^{L} f\left(x, t, u, \frac{\partial u}{\partial t}\right) \psi_{i} d x
\end{aligned}
$$

The last integral in Eq. (13) can be rewritten using Eq. (3) to explicitly account for external, elastic, and viscous forces

$$
\begin{aligned}
\int_{0}^{L} f\left(x, t, u, \frac{\partial u}{\partial t}\right) \psi_{i} d x= & \frac{F_{0}}{A_{0}} \int_{0}^{L} f_{\mathrm{s}}(x, t) \psi_{i} d x \\
& +\sum_{j=1}^{\infty}\left(u_{j} \frac{k_{\mathrm{L}}}{A_{0}} \int_{0}^{L} \gamma_{\mathrm{L}}(x) \psi_{i} \psi_{j} d x\right. \\
& \left.+\frac{\partial u_{j}}{\partial t} \frac{Z_{\mathrm{L}}}{A_{0}} \int_{0}^{L} \zeta_{\mathrm{L}}(x) \psi_{i} \psi_{j} d x\right)
\end{aligned}
$$

To reduce the complexity of the parameter space, Eq. (13) is then nondimensionalized by defining the normalizations $\bar{u}=$ $u / u_{0}, \bar{x}=x / L, \bar{t}=\omega_{0} t, \alpha=E_{\mathrm{m}} / E_{0}, \bar{\zeta}_{\mathrm{L}}(x)=L \zeta_{\mathrm{L}}(x)$, and $\bar{\gamma}_{\mathrm{L}}(x)=L \gamma_{\mathrm{L}}(x)$, where $u_{0}$ is a reference displacement that is defined below and $\omega_{0}$ is the angular frequency of the source. This yields the following dimensionless equations for the modal amplitudes, written as a matrix-vector system:

$$
\ddot{\boldsymbol{U}}(\bar{t})+\bar{Z}_{\mathrm{L}} \mathbf{C} \dot{\boldsymbol{U}}(\bar{t})+\left[\mathbf{K}+\bar{k}_{\mathrm{L}} \boldsymbol{\Gamma}+\alpha \mathbf{K}_{\mathrm{m}}(\bar{t})\right] \boldsymbol{U}(\bar{t})=-\mathbf{F}(\bar{t}),
$$

where $\boldsymbol{U}=\left[\bar{u}_{1}, \bar{u}_{2}, \ldots, \bar{u}_{N}\right]^{\mathrm{T}}, N$ is the number of modes considered in Eq. (7), $\bar{Z}_{\mathrm{L}}=Z_{\mathrm{L}} /\left(A_{0} \rho_{0} c_{0}\right)$ is the ratio of the specific acoustic impedance of the exterior viscous load over the longitudinal wave characteristic acoustic impedance of the beam, $\bar{k}_{\mathrm{L}}=k_{\mathrm{L}} L /\left(E_{0} A_{0}\right)$ is the ratio of the stiffness of the elastic foundation over the effective stiffness of the beam, and $\alpha=E_{\mathrm{M}} / E_{0}$ is the dimensionless modulation amplitude. The components of the matrices in Eq. (15) are

$$
C_{i j}=\frac{c_{0}}{\omega_{0} \beta} \int_{0}^{1} \bar{\zeta}_{\mathrm{L}}(\bar{x}) \psi_{i} \psi_{j} d \bar{x}
$$




$$
\begin{aligned}
K_{i j} & =\left(\frac{\omega_{i}}{\omega_{0}}\right)^{2} \delta_{i j}, \\
\Gamma_{i j} & =\frac{c_{0}^{2}}{\omega_{0}^{2} \beta L} \int_{0}^{1} \bar{\gamma}_{\mathrm{L}}(\bar{x}) \psi_{i} \psi_{j} d \bar{x}, \\
\left(K_{\mathrm{m}}\right)_{i j}(\bar{t}) & =\frac{c_{0}^{2}}{\omega_{0}^{2} \beta L} \int_{0}^{1} \chi(\bar{x}, \bar{t}) \frac{\partial \psi_{i}}{\partial \bar{x}} \frac{\partial \psi_{j}}{\partial \bar{x}} d \bar{x}, \\
F_{i}(\bar{t}) & =\int_{0}^{1} f_{s}(\bar{x}, \bar{t}) \psi_{i} d \bar{x},
\end{aligned}
$$

where $c_{0}=\sqrt{E_{0} / \rho_{0}}$ is the longitudinal wave speed in the beam and $u_{0}$ is chosen such that the coefficient in front of the exterior forcing term is unity. The matrices $\mathbf{C}$ and $\boldsymbol{\Gamma}$ in general have off-diagonal components that couple the modes of the nonmodulated beam unless the elastic and viscous forces are constant throughout the beam. In addition, the matrix $\mathbf{K}_{\mathrm{m}}$ can couple modes at other frequencies, which is further discussed in Sec. II B. Perturbation methods could also be used if $\alpha$ and $v$ are assumed to be small [7,9]. However, we solve Eq. (15) directly in order to find cases of large nonreciprocity for any modulation and material properties of interest.

The initial conditions for Eq. (15) are derived from Eq. (7) and are given by the integral expressions:

$$
\begin{aligned}
& U_{i}(0)=\int_{0}^{L} u(x, 0) \psi_{i} d x, \\
& \dot{U}_{i}(0)=\int_{0}^{L} \dot{u}(x, 0) \psi_{i} d x .
\end{aligned}
$$

Given the system properties, source function, and modulation, Eq. (15) can be solved in the time domain using an ODE solver (e.g., ODE45 solver in MATLAB). However, steady-state solutions can also be sought if the exterior forcing is assumed to be time harmonic, which is detailed in the following section.

\section{B. Steady-state solutions}

Due to the periodicity of $\chi(x, t)$ in time, it can be expanded as a Fourier series,

$$
\chi(\bar{x}, \bar{t})=\sum_{\substack{p=-\infty \\ p \neq 0}}^{\infty} \hat{\chi}^{p}(\bar{x}) e^{-i p v \bar{t}}
$$

where $v=\omega_{\mathrm{m}} / \omega_{0}$ and $i=\sqrt{-1}$. In the remainder of this work, the symbol $i$ refers to the imaginary unit when not used as a subscripted index. We note that the static term $(p=0)$ in Eq. (19) has already been separated in Eq. (5), i.e., $\hat{\chi}^{0}(\bar{x})=0$. We also assume that the source term in Eq. (3) is time harmonic, e.g., $f_{\mathrm{s}}(x, t)=f_{\mathrm{s}}(x) e^{-i \bar{t}}$. As in previous works $[35,36]$, temporal modulation of the waveguide gives rise to harmonics, which are Fourier components of the response that differ from the drive frequency $\omega_{0}$ by integer multiples of the modulation frequency $\omega_{\mathrm{m}}$ (or dimensionless frequencies 1 and $v$, respectively). Thus, we expand the modal amplitudes in the following Fourier series:

$$
\bar{u}_{j}(\bar{t})=\sum_{q=-\infty}^{\infty} \hat{u}_{j}^{q} e^{-i(1+q v) \bar{t}}
$$

where the harmonic mode coefficient $\hat{u}_{j}^{q}$ is the amplitude of the $j$ th mode at the $q$ th harmonic frequency. Substitution of Eq. (20) into Eq. (7) gives the complete solution in terms of the harmonic mode amplitudes,

$$
\bar{u}(\bar{x}, \bar{t})=\sum_{p=-\infty}^{\infty}\left(\sum_{j=1}^{\infty} \hat{u}_{j}^{p} \psi_{j}(\bar{x})\right) e^{-i(1+p v) \bar{t}} .
$$

Substituting Eqs. (19) and (20) into Eq. (15) and utilizing the orthogonality of the Fourier series yields a system of algebraic equations for the harmonic mode amplitudes,

$$
\begin{gathered}
(1+p v)^{2} \hat{\boldsymbol{U}}^{p}+i(1+p v) \bar{Z}_{\mathrm{L}} \mathbf{C} \hat{\boldsymbol{U}}^{p}-\left(\mathbf{K}+\bar{k}_{\mathrm{L}} \boldsymbol{\Gamma}\right) \hat{\boldsymbol{U}}^{p} \\
-\alpha \sum_{q=-P}^{P} \hat{\mathbf{K}}^{p-q} \hat{\boldsymbol{U}}^{q}=\mathbf{F}, \quad p \in[-P, P],
\end{gathered}
$$

where we have truncated the outer summation in Eq. (21) at $p= \pm P$, such that the total number of retained harmonics is $2 P+1, \hat{\boldsymbol{U}}^{p}=\left[\hat{u}_{1}^{p}, \ldots, \hat{u}_{N}^{p}\right]^{\mathrm{T}}$, and

$$
\hat{\mathbf{K}}_{i j}^{p-q}=\frac{c_{0}^{2}}{\omega_{0}^{2} \beta L} \int_{0}^{1} \hat{\chi}^{p-q}(\bar{x}) \frac{\partial \psi_{i}}{\partial \bar{x}} \frac{\partial \psi_{j}}{\partial \bar{x}} d \bar{x} .
$$

\section{Transverse modes}

The derivation of the coupled-mode equations for transverse modes follows an approach similar to what was presented in Secs. II A and II B for the longitudinal mode case. For brevity, only the final equations from the derivation are presented, and the differences between the two approaches are highlighted.

To solve Eq. (2), we seek a solution of the form

$$
w(x, t)=\sum_{i=1}^{\infty} w_{i}(t) \phi_{i}(x),
$$

where $w_{i}(t)$ are the time-dependent modal amplitudes for transverse motion and $\phi_{i}(x)$ are the transverse mode shapes of the nonmodulated beam that satisfy the boundary conditions at each end (clamped, pinned, roller, or free). Following the same procedure as outlined in Secs. II A and II B for the longitudinal wave motion, we find the following equations for the transverse harmonic mode amplitudes:

$$
\begin{gathered}
(1+p v)^{2} \hat{\boldsymbol{W}}^{p}+i(1+p v) \bar{Z}_{\mathrm{T}} \mathbf{C} \hat{\boldsymbol{W}}^{p}-\left(\mathbf{K}+\bar{k}_{\mathrm{T}} \boldsymbol{\Gamma}\right) \hat{\boldsymbol{W}}^{p} \\
-\alpha \sum_{q=-P}^{P} \hat{\mathbf{K}}^{p-q} \hat{\boldsymbol{W}}^{q}=\mathbf{G}, \quad p \in[-P, P],
\end{gathered}
$$

where $\hat{\boldsymbol{W}}^{p}=\left[\hat{w}_{1}^{p}, \ldots, \hat{w}_{N}^{p}\right]^{\mathrm{T}}, \bar{Z}_{\mathrm{T}}=Z_{\mathrm{T}} L /\left(\rho_{0} c_{0} A_{0} R_{\mathrm{g}}\right), \quad \bar{k}_{\mathrm{T}}=$ $k_{\mathrm{T}} L^{3} /\left(E_{0} A_{0} R_{\mathrm{g}}^{2}\right)$, and

$$
\begin{aligned}
C_{i j} & =\frac{c_{0} R_{\mathrm{g}}}{\omega_{0} \beta L} \int_{0}^{1} \bar{\zeta}_{\mathrm{T}}(\bar{x}) \phi_{i} \phi_{j} d \bar{x}, \\
K_{i j} & =\left(\frac{\omega_{i}}{\omega_{0}}\right)^{2} \delta_{i j}, \\
\Gamma_{i j} & =\frac{c_{0}^{2} R_{\mathrm{g}}^{2}}{\omega_{0}^{2} \beta L^{3}} \int_{0}^{1} \bar{\gamma}_{\mathrm{T}}(\bar{x}) \phi_{i} \phi_{j} d \bar{x},
\end{aligned}
$$




$$
\begin{aligned}
\hat{K}_{i j}^{p-q} & =\frac{c_{0}^{2} R_{\mathrm{g}}^{2}}{\omega_{0}^{2} \beta L^{3}} \int_{0}^{1} \hat{\chi}^{p-q}(\bar{x}) \frac{\partial^{2} \phi_{i}}{\partial \bar{x}^{2}} \frac{\partial^{2} \phi_{j}}{\partial \bar{x}^{2}} d \bar{x} \\
G_{i} & =\int_{0}^{1} g_{s}(\bar{x}) \phi_{i} d \bar{x} .
\end{aligned}
$$

Note that since Eq. (2) is a fourth-order PDE, integration by parts is performed twice to yield Eq. (26d). The resulting boundary terms from the integration by parts vanish if the transverse normal modes satisfy clamped, free, pinned, or roller conditions at the boundaries.

\section{EXAMPLE CASE}

The system of equations for the longitudinal and transverse harmonic mode amplitudes, Eqs. (22) and (25), respectively, are general for any modulation forms of $\chi(x, t)$ and any boundary conditions described in Sec. II. Without loss of generality, the present analysis is greatly simplified by considering a fixed-fixed beam for longitudinal modes and a pinnedpinned beam for transverse modes. The mode shapes for longitudinal and transverse vibrations are therefore identical and are written as

$$
\psi_{n}(x)=\phi_{n}(x)=\sin \left(\frac{n \pi x}{L}\right) \quad n=1,2, \ldots, N,
$$

and the natural frequencies are

$$
\omega_{n}=\frac{c_{0} n \pi}{L}
$$

for longitudinal modes and

$$
\omega_{n}=\frac{c_{0} R_{\mathrm{g}} n^{2} \pi^{2}}{L^{2}}
$$

for transverse modes. Consequently, the normalization factor $\beta$ in Eq. (9) is $\beta=L / 2$.

To demonstrate the utility of the models derived in Sec. II, we seek to identify modulation parameters that yield a large degree of nonreciprocity at a point on the beam, $x_{\text {out }}$, given a point force source located at point $x_{\mathrm{s}}$ at the drive frequency $\omega_{0}$. Since the total solution, Eq. (21), is generally aperiodic in time, we focus on only the drive frequency component of the total solution $(p=0)$ in order to investigate the steady-state nonreciprocal energy distribution along the beam. For the present case, we investigate the response of the beam near the resonance frequency of the third mode, $n=3$, which is the lowest frequency that exhibits large nonreciprocity for the modulation parameters considered in Sec. III A. The model geometry is shown in Fig. 2(a) for the longitudinal mode case and Fig. 2(b) for the transverse mode case. A point force is located at $x_{\mathrm{s}}=L / 6$, and the displacement of the beam $u_{\text {out }}$ and $w_{\text {out }}$ is probed at $x_{\text {out }}=5 L / 6$. It is also assumed that there are no exterior elastic forces and the viscous forces are constant throughout the beam, such that mode coupling is induced only by the spatiotemporal modulation of Young's modulus. The exterior forcing functions in Eqs. (3) and (4) then

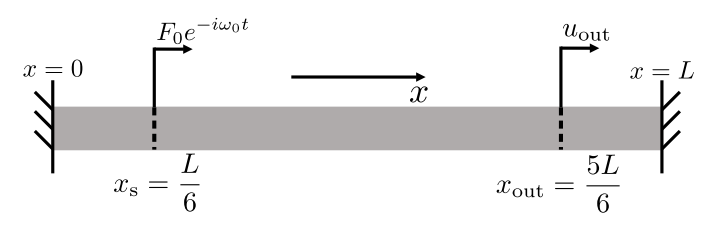

(a)

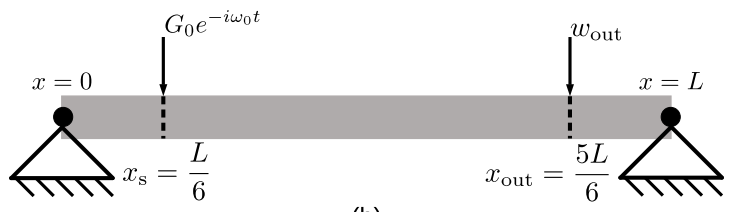

(b)

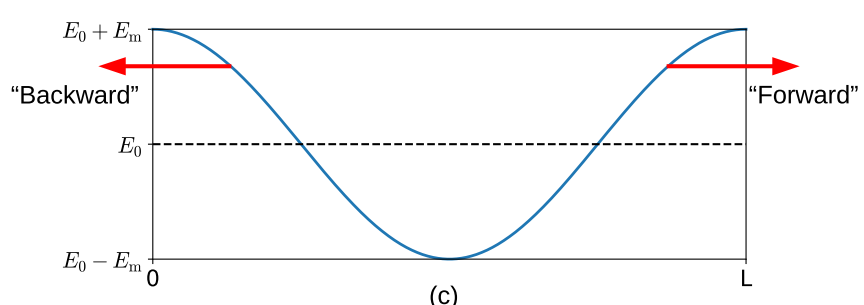

(c)

FIG. 2. Example beam configuration for (a) longitudinal modes with fixed-fixed boundary conditions and (b) transverse modes with pinned-pinned boundary conditions. (c) Plot of Young's modulus at time $t=0$. Nonreciprocity is measured by comparing the displacements $u_{\text {out }}$ and $w_{\text {out }}$ due to time-harmonic sources $F_{0} e^{-i \omega_{0} t}$ and $G_{0} e^{-i \omega_{0} t}$ when the modulation is traveling in the (i) "forward", or $+x$, direction and (ii) "backward", or $-x$, direction.

become

$$
\begin{aligned}
f\left(x, t, \frac{\partial u}{\partial t}\right) & =\frac{F_{0}}{A_{0}} \delta\left(x-x_{\mathrm{s}}\right)+\frac{Z_{\mathrm{L}}}{A_{0}} \frac{\partial u}{\partial t}, \\
g\left(x, t, \frac{\partial w}{\partial t}\right) & =\frac{G_{0}}{A_{0}} \delta\left(x-x_{\mathrm{s}}\right)+\frac{Z_{\mathrm{T}}}{A_{0}} \frac{\partial w}{\partial t},
\end{aligned}
$$

which leads to simplified expressions for the integrals in Eqs. (16a) and (26a),

$$
\int_{0}^{1} \bar{\zeta}_{\mathrm{L}} \psi_{i} \psi_{j} d \bar{x}=\int_{0}^{1} \bar{\zeta}_{\mathrm{T}} \phi_{i} \phi_{j} d \bar{x}=\frac{1}{2} \delta_{i j},
$$

and the exterior forcing vectors in Eqs. (22) and (25),

$$
\begin{gathered}
F_{i}=\psi_{i}\left(x_{\mathrm{s}}\right), \\
G_{i}=\phi_{i}\left(x_{\mathrm{s}}\right) .
\end{gathered}
$$

Young's modulus is modulated with the form

$$
E(x, t)=E_{0}+E_{\mathrm{m}} \cos \left(2 \pi x / L-\omega_{\mathrm{m}} t\right),
$$

as shown in Fig. 2(c), which can be rewritten using complex exponentials such that the mode coupling matrix for longitudinal modes, Eq. (23), becomes

$$
\begin{aligned}
\hat{K}_{m n}^{s}= & \frac{c_{0}^{2} \pi^{2} m n}{\omega_{0}^{2} L^{2}} \\
& \times \begin{cases}\int_{0}^{1} e^{i 2 \pi \bar{x}} \cos (m \pi \bar{x}) \cos (n \pi \bar{x}) d \bar{x}, & s=1, \\
\int_{0}^{1} e^{-i 2 \pi \bar{x}} \cos (m \pi \bar{x}) \cos (n \pi \bar{x}) d \bar{x}, & s=-1, \\
0, & \text { elsewhere, }\end{cases}
\end{aligned}
$$




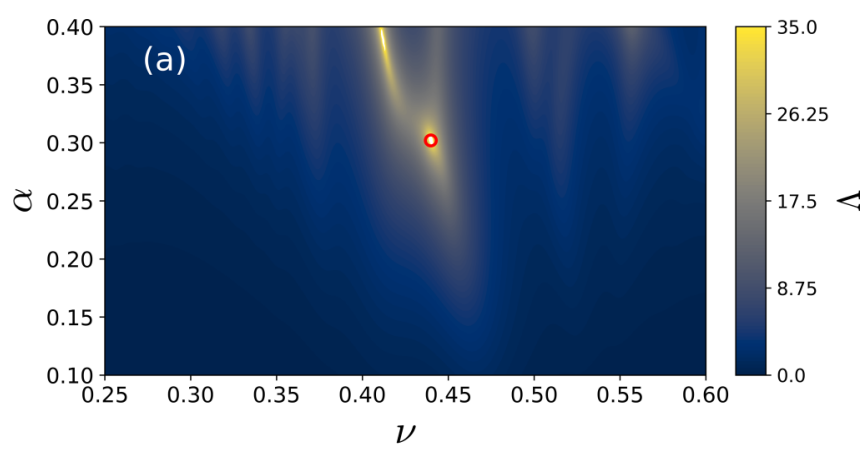

(b)
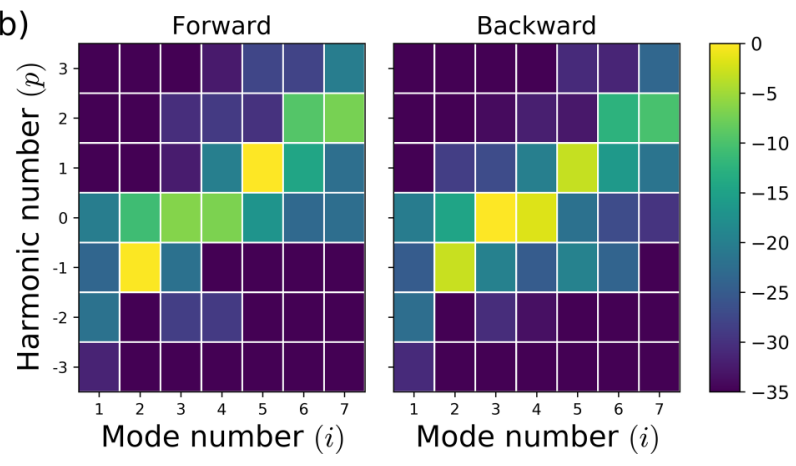

FIG. 3. (a) Nonreciprocity metric $\Lambda$ as a function of $\alpha$ and $v$ for longitudinal modes at $\omega_{0}=3.4 \pi c_{0} / L$ and $\bar{Z}_{\mathrm{L}}=0.5$. The red circle highlights the point $(v, \alpha)=(0.44,0.3)$, which corresponds to the special case considered for (b) and Figs. 4 and 5. (b) Absolute square of the harmonic modal amplitudes, $\left|\hat{u}_{i}^{p}\right|^{2}$, normalized by the absolute square of the maximum harmonic modal amplitude in decibels for the forward and backward modulation directions at $\alpha=0.3$ and $v=0.44$.

and the coupling matrix for transverse modes, Eq. (26d), becomes

$$
\begin{aligned}
\hat{K}_{m n}^{s}= & \frac{c_{0}^{2} R_{\mathrm{g}}^{2} \pi^{4} m^{2} n^{2}}{\omega_{0}^{2} L^{4}} \\
& \times \begin{cases}\int_{0}^{1} e^{i 2 \pi \bar{x}} \sin (m \pi \bar{x}) \sin (n \pi \bar{x}) d \bar{x}, & s=1, \\
\int_{0}^{1} e^{-i 2 \pi \bar{x}} \sin (m \pi \bar{x}) \sin (n \pi \bar{x}) d \bar{x}, & s=-1, \\
0, & \text { elsewhere. }\end{cases}
\end{aligned}
$$

Since the assumed modulation form in Eq. (35) contains only one harmonic term in Eq. (19), only the $s= \pm 1$ terms contribute in Eqs. (36) and (37).

\section{Nonreciprocal vibrations}

The dimensionless parameters in Eqs. (22) and (25) that define the modulation parameter space are the dimensionless modulation amplitude $\alpha$ and the dimensionless modulation frequency $v$. Modulation parameters that yield a large degree of nonreciprocity are sought by performing a grid search within the parameter space with bounds $\alpha \in[0.01,0.8]$ and $v \in[0.01,0.8]$. Due to the geometric symmetry about $x=$ $L / 2$ in Figs. 2(a) and 2(b), reciprocity calculations are carried out by fixing the source and receiver and reversing the direction of the modulation. We first assemble Eqs. (22) and

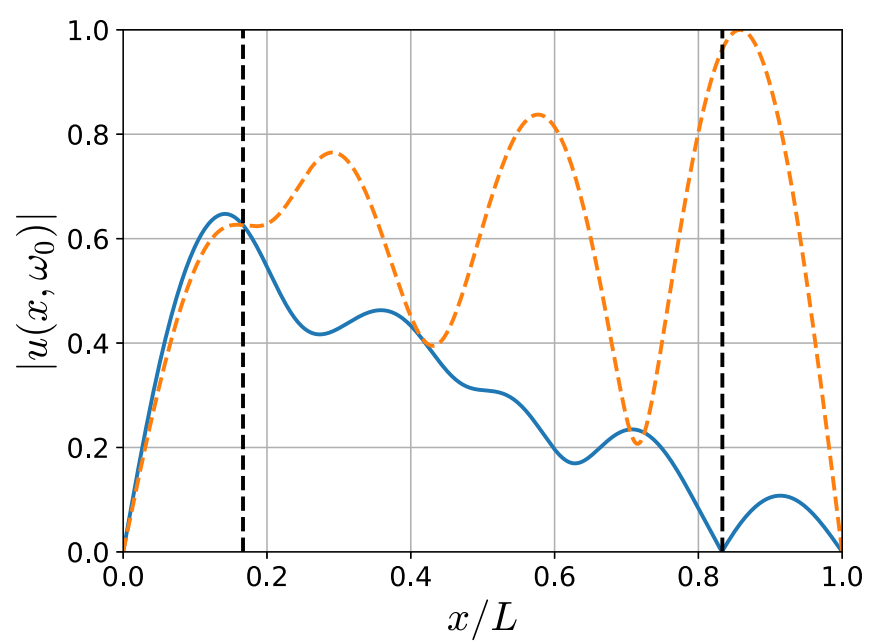

FIG. 4. Magnitude of the normalized longitudinal mode displacement along the beam at the drive frequency $\omega_{0}$ for the forward (blue solid curve) and backward (orange dashed curve) modulation directions at $\alpha=0.3$ and $v=0.44$. The vertical dashed lines at $\bar{x}=1 / 6$ and $\bar{x}=5 / 6$ are the locations of the source and output, respectively.

(25) into a global system of equations and then solve for the case of a Young's modulus that travels in the (i) "forward", or $+x$, direction and (ii) "backward," or $-x$, direction. The displacements $\xi^{+}=u_{\text {out }}, w_{\text {out }}$ at the drive frequency $\omega_{0}$ [the $p=0$ terms in Eq. (21)] for the modulation traveling in the forward direction and the displacements $\xi^{-}=u_{\text {out }}, w_{\text {out }}$
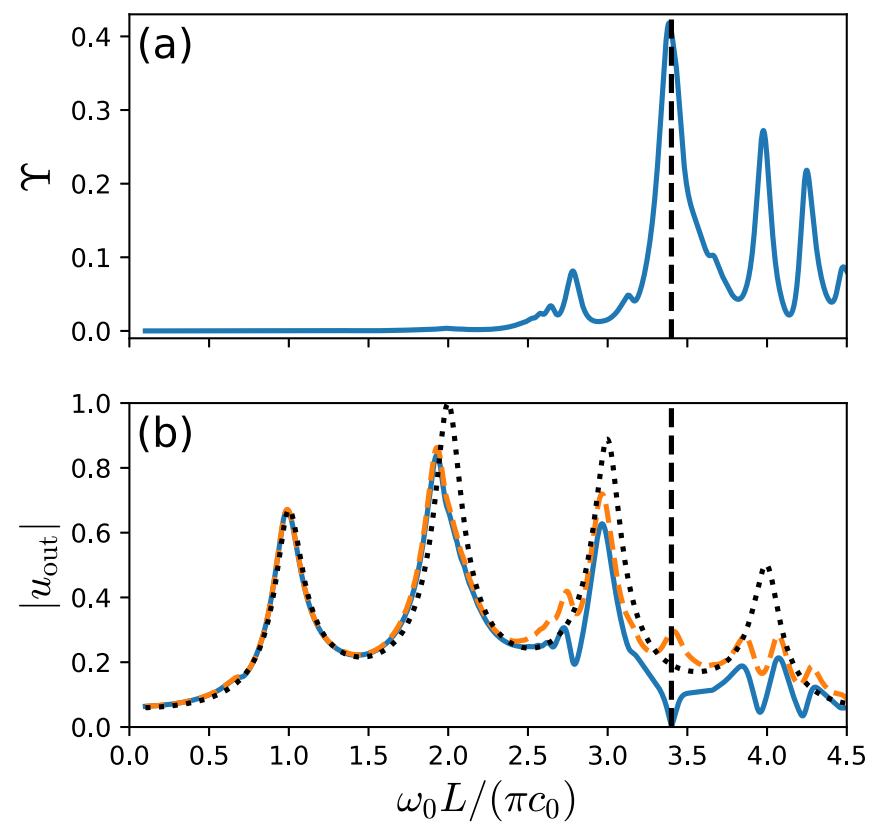

FIG. 5. (a) Nonreciprocity measure $\Upsilon$ as a function of the drive frequency $\omega_{0}$ for the longitudinal mode case at $\alpha=0.3$ and $v=$ 0.44 . (b) $\left|u_{\text {out }}\right|$ as a function of frequency. Blue solid curve: $\xi_{1}$. Orange dashed curve: $\xi_{2}$. Black dotted curve: homogeneous beam with no modulation. The vertical dashed lines in (a) and (b) highlight the drive frequency used in Figs. 3 and 4. 


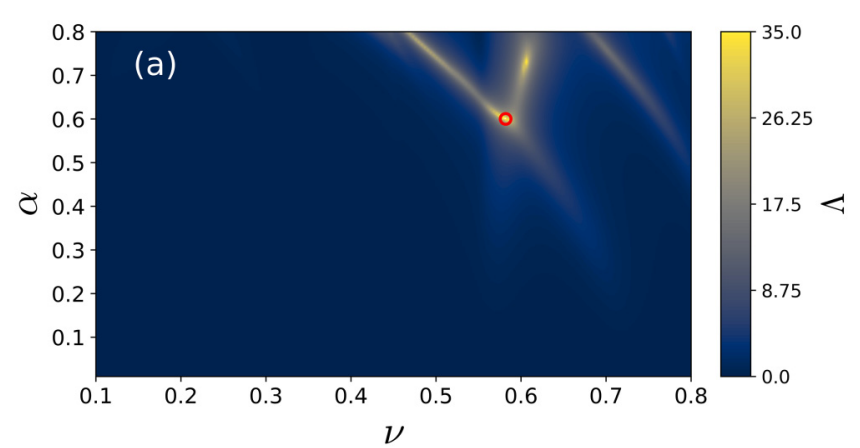

(b)

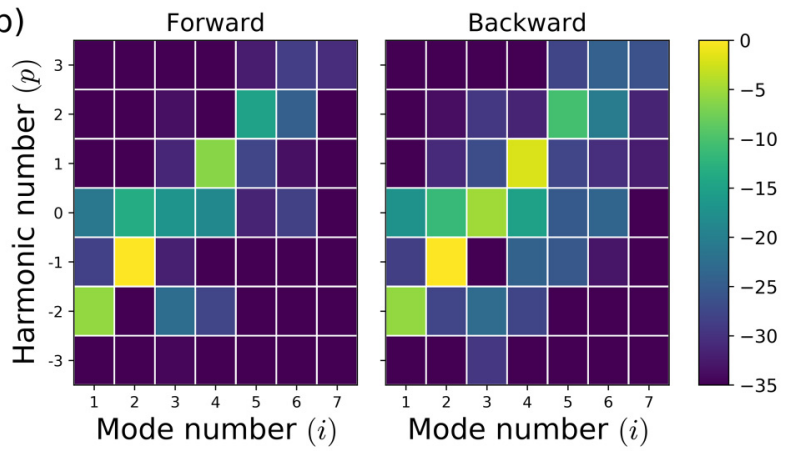

FIG. 6. (a) Nonreciprocity metric $\Lambda$ as a function of $\alpha$ and $v$ for transverse modes at $\omega_{0}=9.288 c_{0} R_{\mathrm{g}} \pi^{2} / L^{2}$ and $\bar{Z}_{\mathrm{T}}=5$. The red circle highlights the point $(v, \alpha)=(0.582,0.6)$, which corresponds to the special case considered for (b) and Figs. 7 and 8. (b) Absolute square of the harmonic modal amplitudes, $\left|\hat{w}_{i}^{p}\right|^{2}$, normalized by the absolute square of the maximum harmonic modal amplitude in decibels for the forward and backward modulation at $\alpha=0.6$ and $v=0.582$.

at the drive frequency $\omega_{0}$ for the modulation traveling in the backward direction are then compared using the following nonreciprocity metric:

$$
\Lambda=\left|20 \log _{10}\left(\left|\frac{\xi^{+}}{\xi^{-}}\right|\right)\right|,
$$

which is zero if the displacements $\xi^{+}, \xi^{-}$are identical (reciprocal) and is nonzero if the system is nonreciprocal.

We first examine the degree of nonreciprocity for longitudinal modes by solving Eq. (22) with the truncation $N=21$ and $P=4$, which was found to be sufficient to resolve the solution at the drive frequency. We set the dimensionless impedance to $\bar{Z}_{\mathrm{L}}=0.5$ to sufficiently damp any strong resonances and harmonic generation effects. The nonreciprocity metric $\Lambda$ is plotted in Fig. 3(a) as a function of $\alpha$ and $v$ for frequency $\omega_{0}=3.4 \pi c_{0} / L$. The modulation parameters that yield a large value of $\Lambda$ form a small subset of the entire parameter space. In addition, this set of modulation parameters contains values of $\alpha$ and $v$ that are not small, which is out of the region of validity for standard coupled-mode perturbation approaches [7,9]. One set of modulation parameters that yields a large $\Lambda$ is $(\nu, \alpha)=(0.44,0.3)$, which is shown in Fig. 3(a) and is used in the longitudinal results detailed below. The absolute square of the harmonic modal amplitudes, $\left|\hat{u}_{i}^{p}\right|^{2}$, normalized by the absolute square of the maximum harmonic mode amplitude in decibels, is shown in Fig. 3(b) for the forward and backward modulation directions. The harmonic

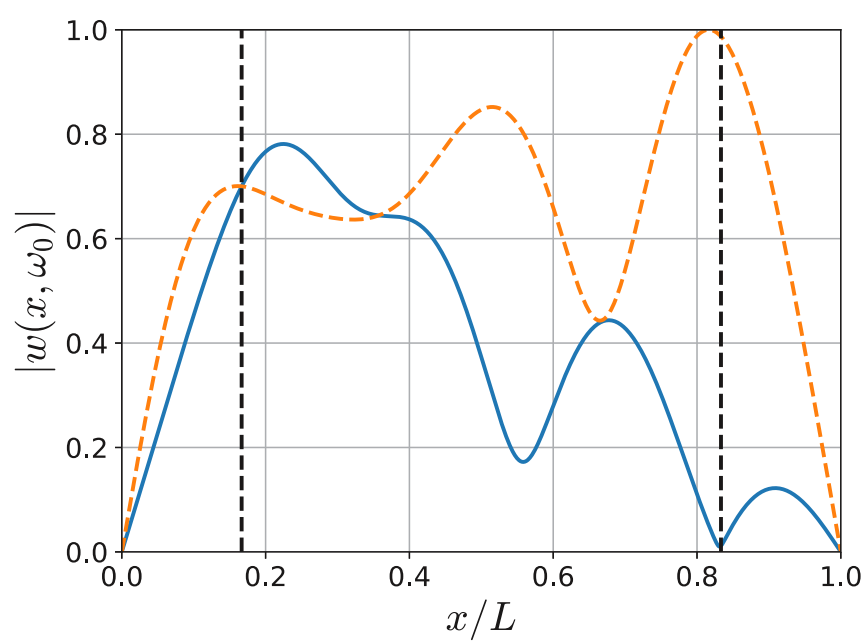

FIG. 7. Magnitude of the normalized transverse mode displacement along the beam at the drive frequency $\omega_{0}$ for the forward (blue solid curve) and backward (orange dashed curve) modulation directions at $\alpha=0.6$ and $v=0.582$. The vertical dashed lines at $\bar{x}=1 / 6$ and $\bar{x}=5 / 6$ are the locations of the source and output, respectively.

modal amplitudes at $p=0$ are different between the forward and backward modulation directions, which yields distinct and therefore nonreciprocal displacement fields between the two modulation directions.

The magnitude of the normalized displacement at the drive frequency $\omega_{0}$ along the beam for the forward and backward
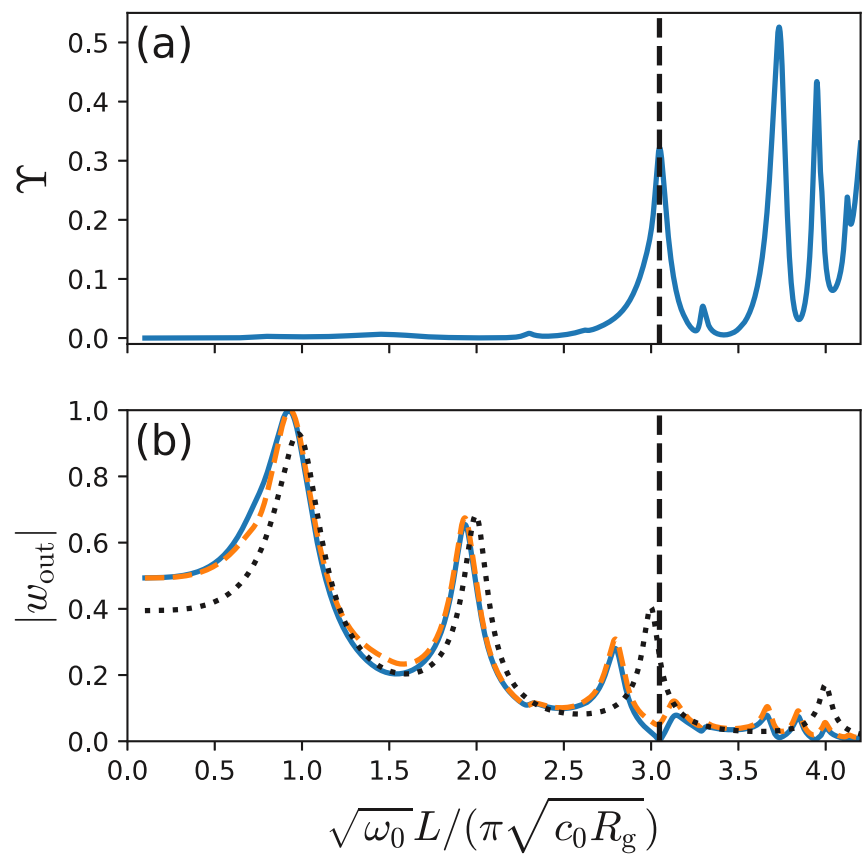

FIG. 8. (a) Nonreciprocity measure $\Upsilon$ as a function of the drive frequency $\omega_{0}$ for the transverse mode case with modulation parameters $\alpha=0.6$ and $v=0.582$. (b) $\left|w_{\text {out }}\right|$ as a function of frequency. Blue solid curve: $\xi_{1}$. Orange dashed curve: $\xi_{2}$. Black dotted curve: homogeneous beam with no modulation. The vertical dashed lines in (a) and (b) highlight the drive frequency used in Figs. 6 and 7. 
modulation directions is plotted in Fig. 4 using the $p=0$ terms in Eq. (21). A null in the displacement field is present for the forward modulation direction at the receiver location, while a large displacement is observed at the receiver location for the backward modulation direction, indicating a very high degree of vibrational nonreciprocity.

We can also characterize the degree of nonreciprocity at all spatial locations along the beam with the following metric:

$$
\Upsilon=\frac{\int_{0}^{L}\left|u^{+}\left(x, \omega_{0}\right)-u^{-}\left(x, \omega_{0}\right)\right|^{2} d x}{\int_{0}^{L}\left|u^{+}\left(x, \omega_{0}\right)\right|^{2} d x+\int_{0}^{L}\left|u^{-}\left(x, \omega_{0}\right)\right|^{2} d x},
$$

where $u^{ \pm}\left(x, \omega_{0}\right)$ are the two solutions of Eq. (21) for the forward and backward modulation directions at the drive frequency $\omega_{0}$, respectively. The metric $\Upsilon$ is zero if the system is reciprocal everywhere along the beam and increases in value as more locations along the beam exhibit nonreciprocity. This metric is shown as a function of drive frequency in Fig. 5(a), and the displacements $\xi^{+}$and $\xi^{-}$along with the displacement of the nonmodulated beam at $x_{\text {out }}$ as a reference case are shown in Fig. 5(b). A small degree of nonreciprocity is observed for frequencies near the first and second modes and increases for frequencies near the third and fourth modes.

Finally, the degree of nonreciprocity for transverse modes is explored by plotting $\Lambda$ in Fig. 6(a) as a function of $\alpha$ and $v$ for $\omega_{0}=9.288 c_{0} R_{\mathrm{g}} \pi^{2} / L^{2}$ and $\bar{Z}_{\mathrm{T}}=5$. Note that, for this drive frequency, larger values of $\alpha$ and $v$ are needed to obtain large values of $\Lambda$ compared to the longitudinal case in Fig. 3(a). A modulation parameter set that obtains a large value of $\Lambda$ is highlighted in Fig. 6(a) with the values $(v, \alpha)=$ $(0.582,0.6)$ and is used in the transverse mode results detailed below. The absolute square of the harmonic modal amplitudes $\left|\hat{w}_{i}^{p}\right|^{2}$ normalized by the absolute square of the maximum harmonic modal amplitude in decibels is shown in Fig. 6(b) for the forward and backward modulation directions. Note that this case exhibits stronger harmonic generation than in the longitudinal case. The magnitude of the transverse displacement at the drive frequency along the beam for both the forward and backward modulation directions is plotted in Fig. 7. As in the longitudinal mode case, a null in the transverse displacement is present at the receiver location for the forward modulation direction, while a large displacement is observed at the receiver location for the backward modulation case. Finally, we plot $\Upsilon$ as a function of the drive frequency in Fig. 8(a) and the displacements $\xi^{+}$and $\xi^{-}$along with the transverse displacement of the nonmodulated beam at $x_{\text {out }}$ as a reference solution in Fig. 8(b). While the degree of nonreciprocity is less than in the case of longitudinal modes in Fig. 5(a), nonreciprocity can still be observed at drive frequencies near the resonance frequency of the third and fourth modes.

\section{CONCLUSION}

We have developed a semianalytical coupled-mode model to study nonreciprocal longitudinal and transverse vibrations in a finite Euler-Bernoulli beam with a Young's modulus that is modulated in space and time. The method presented in this work exactly satisfies the boundary conditions by using the mode shapes of the nonmodulated beam as a basis set and converges to the exact solution when sufficient numbers of modes and frequency harmonics are retained in the solution for the harmonic modal amplitudes. No assumptions were made about the magnitudes of the values of the modulation strength and frequency, which enabled a complete exploration of the modulation parameter space for designs that yield a high degree of nonreciprocity. To facilitate the exploration of the design space, we identified a small set of dimensionless parameters that characterize the relative strengths of the modulation and dissipation.

For the cases considered in this work, we have demonstrated that this system displays a large degree of nonreciprocity for a small subset of modulation parameters for both lowfrequency longitudinal and transverse modes, most of which contain values of the modulation parameters that are outside the region of applicability for perturbation theory approaches. The present coupled-mode approach can be generalized to more complex systems, such as coupled waveguides and multiple spatial dimensions. In this case, a numerical technique such as the finite-element method (FEM) [43] can be utilized to generate a complete orthogonal set of basis functions via the associated eigenvalue problem. Additionally, the integral statements in Eqs. (16a)-(16e) provide a natural setting for using the mode shapes obtained from FEM. Modeling, design, and experimental efforts should be explored to find optimized modulation and system parameters that yield a large degree of nonreciprocity; to elucidate the role of material losses in the degree of nonreciprocity; and to generate broadband nonreciprocal behavior, rather than at the drive frequency only.

\section{ACKNOWLEDGMENTS}

This work was supported by National Science Foundation EFRI Award No. 1641078 and the Postdoctoral Fellowship Program at Applied Research Laboratories at The University of Texas at Austin.
[1] J. W. Strutt, Some general theorems relating to vibrations, Proc. London Math Soc. s1-4, 357 (1871).

[2] J. Achenbach, Reciprocity in Elastodynamics (Cambridge University Press, Cambridge, 2003).

[3] H. B. G. Casimir, On Onsager's principle of microscopic reversibility, Rev. Mod. Phys. 17, 343 (1945).

[4] K. Yi, M. Collet, and S. Karkar, Frequency conversion induced by time-space modulated media, Phys. Rev. B 96, 104110 (2017)
[5] M. B. Zanjani, A. R. Davoyan, A. M. Mahmoud, N. Engheta, and J. R. Lukes, One-way phonon isolation in acoustic waveguides, Appl. Phys. Lett. 104, 081905 (2014).

[6] G. Trainiti and M. Ruzzene, Non-reciprocal elastic wave propagation in spatiotemporal periodic structures, New J. Phys. 18, 083047 (2016).

[7] H. Nassar, H. Chen, A. N. Norris, M. R. Haberman, and G. L. Huang, Non-reciprocal wave propagation in modulated elastic metamaterials, Proc. R. Soc. A 473, 20170188 (2017). 
[8] R. Fleury, D. L. Sounas, C. F. Sieck, M. R. Haberman, and A. Alù, Sound isolation and giant linear nonreciprocity in a compact acoustic circulator, Science 343, 516 (2014).

[9] R. Fleury, D. L. Sounas, and A. Alù, Subwavelength ultrasonic circulator based on spatiotemporal modulation, Phys. Rev. B 91, 174306 (2015).

[10] R. Fleury, A. B. Khanikaev, and A. Alu, Floquet topological insulators for sound, Nat. Commun. 7, 11744 (2016).

[11] Y. Ding, Y. Peng, Y. Zhu, X. Fan, J. Yang, B. Liang, X. Zhu, X. Wan, and J. Cheng, Experimental Demonstration of Acoustic Chern Insulators, Phys. Rev. Lett. 122, 014302 (2019).

[12] R. Fleury, D. Sounas, M. R. Haberman, and A. Alu, Nonreciprocal acoustics, Acoust. Today 11, 14 (2015).

[13] M. R. Haberman and M. D. Guild, Acoustic metamaterials, Phys. Today 69(6), 42 (2016).

[14] A. Merkel, M. Willatzen, and J. Christensen, Dynamic Nonreciprocity in Loss-Compensated Piezophononic Media, Phys. Rev. Appl. 9, 034033 (2018).

[15] O. A. Godin, Reciprocity and energy theorems for waves in a compressible inhomogeneous moving fluid, Wave Motion 25, 143 (1997).

[16] O. A. Godin, Recovering the Acoustic Green's Function From Ambient Noise Cross Correlation in an Inhomogeneous Moving Medium, Phys. Rev. Lett. 97, 054301 (2006).

[17] L. M. Nash, D. Kleckner, A. Read, V. Vitelli, A. M. Turner, and W. T. M. Irvine, Topological mechanics of gyroscopic metamaterials, Proc. Natl. Acad. Sci. USA 112, 14495 (2015).

[18] P. Wang, L. Lu, and K. Bertoldi, Topological Phononic Crystals with One-Way Elastic Edge Waves, Phys. Rev. Lett. 115, 104302 (2015).

[19] Y. Chen, X. Li, H. Nassar, A. N. Norris, C. Daraio, and G. Huang, Nonreciprocal Wave Propagation in a ContinuumBased Metamaterial with Space-Time Modulated Resonators, Phys. Rev. Appl. 11, 064052 (2019).

[20] C. Shen, X. Zhu, J. Li, and S. A. Cummer, Nonreciprocal acoustic transmission in space-time modulated coupled resonators, Phys. Rev. B 100, 054302 (2019).

[21] M. D. Fronk, S. Tawfick, C. Daraio, S. Li, A. Vakakis, and M. J. Leamy, Acoustic non-reciprocity in lattices with nonlinearity, internal hierarchy, and asymmetry: Computational study, J. Vib. Acoust. 141, 051011 (2019).

[22] Z. Zhang, I. Koroleva, L. I. Manevitch, L. A. Bergman, and A. F. Vakakis, Nonreciprocal acoustics and dynamics in the in-plane oscillations of a geometrically nonlinear lattice, Phys. Rev. E 94, 032214 (2016).

[23] J. Bunyan, K. J. Moore, A. Mojahed, M. D. Fronk, M. Leamy, S. Tawfick, and A. F. Vakakis, Acoustic nonreciprocity in a lattice incorporating nonlinearity, asymmetry, and internal scale hierarchy: Experimental study, Phys. Rev. E 97, 052211 (2018).

[24] Z. Lu and A. N. Norris, Non-reciprocal wave transmission in a bilinear spring-mass system, J. Vib. Acoust. 142, 021006 (2020)

[25] E. Cassedy and A. Oliner, Dispersion relations in time-space periodic media: Part I-Stable interactions, Proc. IEEE 51, 1342 (1963).

[26] E. Cassedy, Dispersion relations in time-space periodic media: Part II-Unstable interactions, Proc. IEEE 55, 1154 (1967).
[27] J. Vila, R. K. Pal, M. Ruzzene, and G. Trainiti, A Bloch-based procedure for dispersion analysis of lattices with periodic timevarying properties, J. Sound Vib. 406, 363 (2017).

[28] H. Nassar, X. C. Xu, A. N. Norris, and G. L. Huang, Modulated phononic crystals: Non-reciprocal wave propagation and Willis materials, J. Mech. Phys. Solids 101, 10 (2017).

[29] H. Nassar, H. Chen, A. N. Norris, and G. L. Huang, Quantization of band tilting in modulated phononic crystals, Phys. Rev. B 97, 014305 (2018).

[30] X. Zhu, J. Li, C. Shen, X. Peng, A. Song, L. Li, and S. A. Cummer, Non-reciprocal acoustic transmission via spacetime modulated membranes, Appl. Phys. Lett. 116, 034101 (2020).

[31] C. Croënne, J. Vasseur, O. Bou Matar, M.-F. Ponge, P. A. Deymier, A.-C. Hladky-Hennion, and B. Dubus, Brillouin scattering-like effect and non-reciprocal propagation of elastic waves due to spatio-temporal modulation of electrical boundary conditions in piezoelectric media, Appl. Phys. Lett. 110, 061901 (2017).

[32] A. Nanda and M. A. Karami, One-way sound propagation via spatio-temporal modulation of magnetorheological fluid, J. Acoust. Soc. Am. 144, 412 (2018).

[33] M. Ansari, M. Attarzadeh, M. Nouh, and M. A. Karami, Application of magnetoelastic materials in spatiotemporally modulated phononic crystals for nonreciprocal wave propagation, Smart Mater. Struct. 27, 015030 (2017).

[34] Y. Wang, B. Yousefzadeh, H. Chen, H. Nassar, G. Huang, and C. Daraio, Observation of Nonreciprocal Wave Propagation in a Dynamic Phononic Lattice, Phys. Rev. Lett. 121, 194301 (2018).

[35] S. P. Wallen and M. R. Haberman, Nonreciprocal wave phenomena in spring-mass chains with effective stiffness modulation induced by geometric nonlinearity, Phys. Rev. E 99, 013001 (2019).

[36] B. M. Goldsberry, S. P. Wallen, and M. R. Haberman, Nonreciprocal wave propagation in mechanically-modulated continuous elastic metamaterials, J. Acoust. Soc. Am. 146, 782 (2019).

[37] H. Nassar, H. Chen, A. N. Norris, and G. L. Huang, Nonreciprocal flexural wave propagation in a modulated metabeam, Extreme Mech. Lett. 15, 97 (2017).

[38] M. Attarzadeh and M. Nouh, Non-reciprocal elastic wave propagation in $2 \mathrm{D}$ phononic membranes with spatiotemporally varying material properties, J. Sound Vib. 422, 264 (2018).

[39] E. Riva, M. Di Ronco, A. Elabd, G. Cazzulani, and F. Braghin, Non-reciprocal wave propagation in discretely modulated spatiotemporal plates, J. Sound Vib. 471, 115186 (2020).

[40] G. Trainiti, Y. Xia, J. Marconi, G. Cazzulani, A. Erturk, and M. Ruzzene, Time-Periodic Stiffness Modulation in Elastic Metamaterials for Selective Wave Filtering: Theory and Experiment, Phys. Rev. Lett. 122, 124301 (2019).

[41] J. Marconi, E. Riva, M. Di Ronco, G. Cazzulani, F. Braghin, and M. Ruzzene, Experimental Observation of Nonreciprocal Band Gaps in a Space-Time-Modulated Beam Using a Shunted Piezoelectric Array, Phys. Rev. Appl. 13, 031001 (2020).

[42] L. Meirovitch, Fundamentals of Vibrations (Waveland, Long Grove, Illinois, 2010).

[43] M. S. Gockenbach, Understanding and Implementing the Finite Element Method (SIAM, Philadelphia, 2006). 